\title{
Bio-templated Multilayered Organic-Inorganic Composites Investigated by Analytical STEM
}

\author{
Vesna Srot ${ }^{1}$, Pouya Moghimian ${ }^{1}$, Sandra Facey $^{2}$ and Peter A. van Aken ${ }^{1}$ \\ 1. Stuttgart Center for Electron Microscopy, Max Planck Institute for Solid State Research, Stuttgart, \\ Germany. \\ 2. Institute of Technical Biochemistry, University of Stuttgart, Germany.
}

Bio-inspired synthesis of organic-inorganic hybrid structures at moderate temperatures is one of the most promising areas in bio-nanotechnology. Inorganic nanomaterials are templated by organic components, which results in a broad range of applications for the resulted structures in the fields of organic electronics and photonics [1]. Various mechanisms can trigger the nucleation and consequent growth of hybrid organic-inorganic nanostructures, where the organic components are considered as the scaffolds [2].

Wild-type (WT) M13 phages have been widely used in biotechnology as a biomimetic matrix for tissue engineering and as biological templates for materials design. The M13 phage is a filamentous virus with an elongated shape, possessing capability of self-assembling into liquid crystal structures, providing modifiable surfaces for the fabrication of hybrid structures.

For our study we employed M13 phages as scaffolds for assembling layer-by-layer structures consisting of alternating M13 and $\mathrm{ZnO}$ layers. We used an in-house build convective assembly apparatus to construct directionally oriented M13-WT phage layers deposited on C-coated Si wafers. Our previous studies showed beneficial effects to use an amorphous carbon (a-C) surface for directional alignment of the M13 phages [3,4].

Bright-field (BF-) and high-angle annular dark-field (HAADF-) scanning transmission electron microscopy (STEM) images (Figure 1a and 1b) of a cross-section of the multilayered sample consisting of alternating five thin layers of M13 phages and five $\mathrm{ZnO}$ layers, which were assembled on an a-C sputtered Si substrate. A HAADF-STEM image at higher magnification of the first M13 phage layer followed by the $\mathrm{ZnO}$ layer is shown in Figure 1c. The high-resolution transmission electron microscopy (HRTEM) image (Figure 1d) displays a phage layer between two nanocrystalline ZnO layers.

The chemical identity of the multilayers was investigated by electron energy-loss spectroscopy (EELS). Spectrum images (SI) were acquired from the M13-ZnO interfaces as shown in the HAADF-STEM image (Figure 2a). Corresponding $\mathrm{C}-\mathrm{K}, \mathrm{O}-\mathrm{K}$ and $\mathrm{Zn}-\mathrm{L}_{2,3}$ spectrum images after background subtraction are shown in Figures $2 \mathrm{~b}-\mathrm{d}$. A homogeneous organic-inorganic multilayered structure with only minor thickness fluctuation and with limited interpenetration at the M13-ZnO interfaces was achieved. Our work can serve as a foundation for future bio-templated organic-inorganic multilayered structures with controlled ratios between organic and inorganic components.

\section{References:}

[1] L Shen et al., J Mater Chem 21 (2011), 18868-76.

[2] M Habib et al., Life Robotics 17 (2012), 191-196.

[3] P Moghimian et al., Langmuir 30 (2014), 11428-32.

[4] P Moghimian et al., Int J of Mater Research 107 (2016), 295-299. 

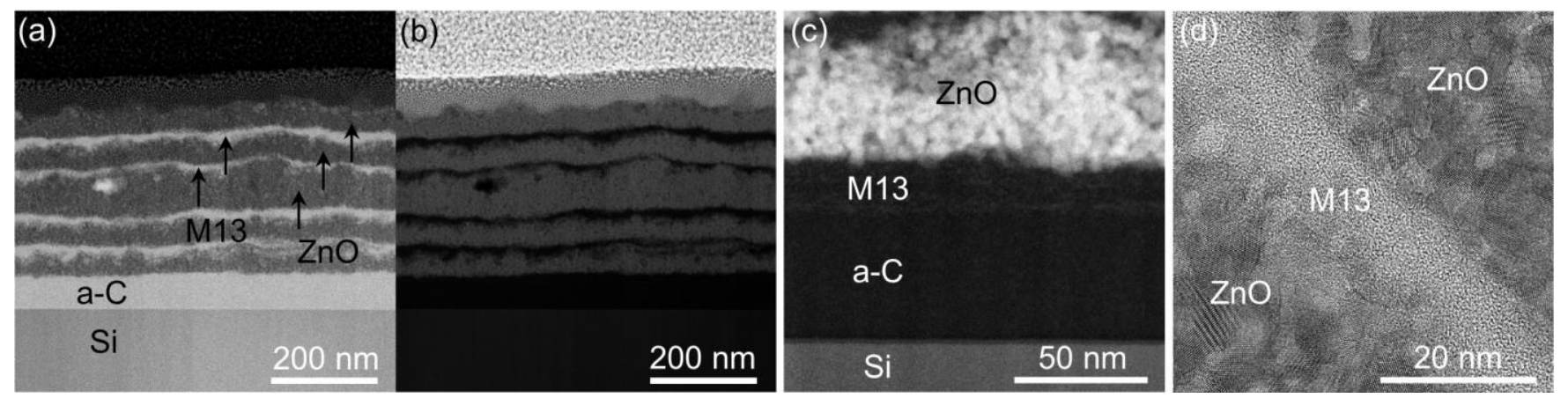

Figure 1. (a, b) BF- and HAADF-STEM images of a cross-section of the organic-inorganic multilayered structure consisting of alternating M13 phage and ZnO layers deposited on an a-C sputtered Si substrate. (c) HAADF-STEM image at higher magnification of the first M13 layer followed by a ZnO layer. (d) HRTEM image of a M13 layer between two consecutive ZnO layers.

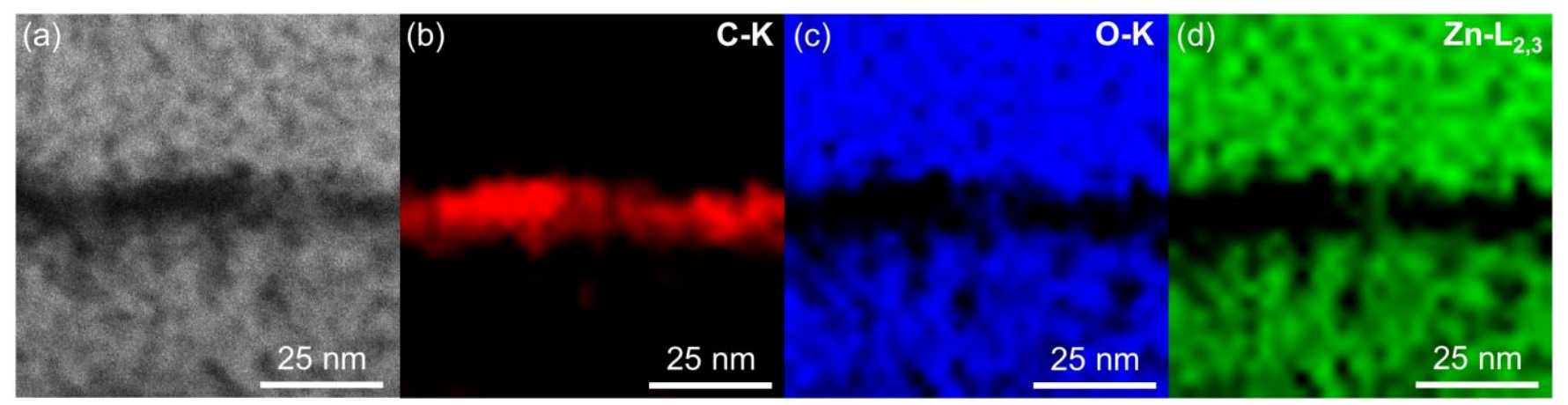

Figure 2. (a) HAADF-STEM image of a M13 layer between two ZnO layers with corresponding (b) C$\mathrm{K}$, (c) $\mathrm{O}-\mathrm{K}$ and (d) $\mathrm{Zn}-\mathrm{L}_{2,3}$ EELS spectrum images. 